\title{
Further contributions to the aleocharine fauna of the Yukon Territory, Canada (Coleoptera, Staphylinidae)
}

\author{
Jan Klimaszewski ${ }^{1, \dagger}$, Benoit Godin,$\ddagger$, Caroline Bourdon ${ }^{1, \S}$ \\ I Natural Resources Canada, Canadian Forest Service, Laurentian Forestry Centre, 1055 du P.E.P.S., P.O. \\ Box 10380, Stn. Sainte-Foy, Québec, Quebec, Canada G1V 4 C7 2 Environment Canada, 91782 Alaska \\ Highway, Whitehorse, Yukon, Canada Y1A $5 B 7$ \\ † urn:lsid:zoobank.org:author:75880C14-430B-45F6-8B6D-840428F3FF37 \\ ¥ urn:lsid:zoobank.org:author:8EB1A90E-239F-4BD3-8DC6-7E7F509C1B96 \\ § urn:lsid:zoobank.org:author:BF4FC97D-284F-449E-9D6A-836846CCBFE6 \\ Corresponding author: Jan Klimaszewski (jan.klimaszewski@nrcan-rncan.gc.ca)
}

Academic editor: C. Majka | Received 13 January 2012 | Accepted 6 March 2012 | Published 26 April 2012

urn:lsid:zoobank.org:pub:2C1D104B-80A9-4066-B5DC-7C815845BB89

Citation: Klimaszewski J, Godin B, Bourdon C (2012) Further contributions to the aleocharine fauna of the Yukon Territory, Canada (Coleoptera, Staphylinidae). In: Klimaszewski J, Anderson R (Eds) Biosystematics and Ecology of Canadian Staphylinidae (Coleoptera) II. ZooKeys 186: 207-237. doi: 10.3897/zookeys.186.2674

\begin{abstract}
The aleocharine beetles of the Yukon Territory, Canada are reviewed based on material studied since the most recent survey of the territory in 2008. The present contribution recognizes a fauna of 125 species, of which 9 are new to science, 20 represent new territorial records and one represents a new Canadian record. Seventeen species are considered Holarctic, 6 introduced, and 2 species are of undetermined status (Holarctic or adventive). The Yukon fauna is classified in 32 genera and 8 tribes. The new species are: 1) Acrotona horwoodae Klimaszewski \& Godin, sp. n.; 2) Atheta (Microdota) microelytrata Klimaszewski \& Godin, sp. n.; 3) Atheta (Microdota) riparia Klimaszewski \& Godin, sp. n.; 4) Atheta (Datomicra) whitehorsensis Klimaszewski \& Godin, sp. n.; 5) Ocyusa yukonensis Klimaszewski \& Godin, sp. n.; 6) Philhygra pseudolarsoni Klimaszewski \& Godin, sp. n.; 7) Philhygra terrestris Klimaszewski \& Godin, sp. n.; 8) Boreophilia davidgei Klimaszewski \& Godin, sp. n.; and 9) Boreophilia herschelensis Klimaszewski \& Godin, sp. n.
\end{abstract}

\section{Keywords}

Canada, Coleoptera, Staphylinidae, Aleocharinae, taxonomy, Yukon

Copyright Her Majesty the Queen in Right of Canada. This is an open access article distributed under the terms of the Creative Commons Attribution License 3.0 (CC-BY), which permits unrestricted use, distribution, and reproduction in any medium, provided the original author and source are credited. 


\section{Introduction}

Aleocharinae is the largest subfamily of Staphylinidae and embraces a wide variety of morphologically and ecologically diverse species that are poorly documented in Canada. This subfamily is widely distributed in North America and occurs in almost all terrestrial habitats. Most species are found in forests where they occur in leaf litter, under bark, in fungi, in moss and within the nests of ants, mammals and birds. In forest litter, the aleocharine fauna is a dominant group and part of a complex ecological web that is responsible for nutrient cycling, which ultimately contributes to forest productivity and resilience (Buse and Good 1993, Leschen 1993).

Currently, over 400 species of Aleocharinae in 92 genera are recorded from Canada and Alaska (Gouix and Klimaszewski 2007, Webster et al. 2009, Majka and Klimaszewski 2010, Klimaszewski et al. 2011). In a checklist of Canadian Coleoptera, Campbell and Davies (1991) recorded 59 species of Aleocharinae from the Yukon Territory. Gouix and Klimaszewski (2007) reported a fauna of 65 aleocharine species and in a more focused study of Yukon material, Klimaszewski et al. (2008) described 6 new species and provided 24 new territorial records, raising the total number of species to 95 .

The present paper provides an updated review of aleocharine beetles from the $\mathrm{Yu}-$ kon Territory and constitutes important baseline data for monitoring the impact of invasive species, pollution, natural resource extraction and climate change. Additionally, the information and illustrations contained herein will make it possible to incorporate this diverse subfamily into ongoing Canadian biodiversity inventories including those in the Canadian Arctic.

\section{Materials and methods}

Over 1,226 adults of Aleocharinae from the Yukon Territory were studied and most specimens were dissected to examine genitalia. The genital structures were dehydrated in absolute alcohol, mounted in Canada balsam on celluloid microslides and pinned with the specimens from which they originated. Photographs of the entire body and the genital structures were taken using an image processing system (Nikon SMZ 1500 stereoscopic microscope; Nikon Digit-like Camera DXM 1200F) and Adobe Photoshop software.

Morphological terminology mainly follows that used by Seevers (1978), Klimaszewski (1984) and Ashe (2001). The ventral part of the median lobe of the aedeagus is considered to be the part of the bulbus containing the foramen mediale, the entrance of the ductus ejaculatorius and the adjacent ventral part of the tubus of the median lobe with an internal sac and its structures (this part is referred to as the parameral side in some recent publications); the opposite side is referred to as dorsal. In the species descriptions, microsculpture refers to the surface of the upper forebody (head, pronotum and elytra). 
Samples collected in this study include those from the Ecological Monitoring and Assessment Network (EMAN) plots. Two 1 ha plots, the Fireweed Drive (mixed pine and willow forest) and Cadet Camp (white spruce mature forest with feathermoss ground cover), have been reserved for long-term monitoring. All samples from these locations were collected from pitfall traps operating from late May to late September. Additional pitfall samples were collected by Donald Reid from early June to early August 2007, and early June to mid August 2008 at an alluvial fan on Hershel Island (dominated by Carex and grasses with some willows). All other sample collections were from organic litter sifting.

\section{Depository/institutional abbreviations:}

CNC Canadian National Collection of Insects, Arachnids and Nematodes, Agriculture and Agri-Food Canada, Ottawa, Ontario, Canada

ECW Environment Canada, Whitehorse, Yukon, Canada

LFC Natural Resources Canada, Canadian Forest Service, Laurentian Forestry Centre, René Martineau Insectarium, Québec City, Quebec, Canada

\section{Results}

In this second recent survey of the Aleocharinae of the Yukon Territory, 125 species in 32 genera and 8 tribes are reported, including two tentative records. Nine species are newly described herein, 20 additional species constitute new territorial records and one species represents a new Canadian record. There are 6 adventive and 17 Holarctic species known from the territory and the status of two other species cannot yet be determined as belonging to either category. Adventive species constitute $4.8 \%$ of the total known aleocharine fauna of the Yukon.

\section{Discussion}

The present survey increased the known Yukon aleocharine fauna from 95 to 125 species (Klimaszewski et al. 2008) and represents a significant contribution to the documentation of Canada's entomofauna. Recent baseline surveys of Aleocharinae in other regions of Canada reported 203 species from the Maritime Provinces of Canada, of which 174 have been recorded in the past decade (Majka and Klimaszewski 2010), and 172 species from Newfoundland and Labrador (Klimaszewski et al. 2011).

Intensive sampling of the aleocharine fauna of the Yukon is continuing by the second author and undoubtedly many more species will be discovered in the future. The study of the Yukon fauna is particularly significant for understanding the shift in some species distributions in response to climate warming and for establishing baseline 
biodiversity data for northern Canada. Additionally, the occurrence of a species in the Yukon Territory otherwise known only from the eastern part of the country provides some evidence for a natural Holarctic distribution. Therefore, a survey of the biodiversity of the Yukon also contributes to our knowledge of species suspected of being adventive.

\section{Checklist of Aleocharinae species in the Yukon Territory}

(* adventive species, ${ }^{* *}$ Holarctic species, NTR=new territorial record for the Yukon Territory, NCR=new Canadian record; taxa in phylogenetic order).

\section{Order Coleoptera}

\section{Family Staphylinidae Latreille}

Subfamily Aleocharinae Fleming

I. Tribe Gymnusini Heer

\section{Gymnusa Gravenhorst}

Brevicollis Group

1. Gymnusa atra Casey**

2. Gymnusa konopackii Klimaszewski

Variegata Group

3. Gymnusa pseudovariegata Klimaszewski

4. Gymnusa smetanai Klimaszewski**

5. Gymnusa campbelli Klimaszewski

II. Tribe Aleocharini Fleming

\section{Aleochara Gravenhorst}

Subgenus Aleochara s. str.

6. Aleochara (s. str.) assiniboin Klimaszewski

7. Aleochara (s. str.) lata Gravenhorst*

8. Aleochara (s. str.) sekanai Klimaszewski

9. Aleochara (s. str.) tahoensis Casey

Subgenus Coprochara

10. Aleochara (Coprochara) verna Say

Subgenus Xenochara

11. Aleochara (Xenochara) castaneipennis Mannerheim

12. Aleochara (Xenochara) fumata Gravenhorst*

III. Tribe Oxypodini Thomson

\section{Calodera Mannerheim}

13. Calodera parviceps (Casey) (NTR)

Devia Blackwelder 
14. Devia prospera (Erichson)**

\section{Gnathusa Fenyes}

15. Gnathusa caribou Lohse

16. Gnathusa eva Fenyes (NTR)

17. Gnathusa tenuicornis Fenyes (NTR)

\section{Parocalea Bernhauer}

18. Parocalea nearctica Lohse

19. Parocalea pseudobaicalica Lohse

\section{Neothetalia Klimaszewski}

20. Neothetalia canadiana Klimaszewski

\section{Ocyusa Kraatz}

21. Ocyusa yukonensis Klimaszewski \& Godin, sp. n.

22. Ocyusa canadensis Lohse

\section{Oxypoda Mannerheim}

Convergens Group

23. Oxypoda pseudoconvergens Klimaszewski \& Godin

24. Oxypoda canadensis Klimaszewski (NTR)

Lacustris Group

25. Oxypoda lacustris Casey

26. Oxypoda hiemalis Casey

Lucidula Group

27. Oxypoda lucidula Casey

28. Oxypoda demissa Casey

Operta Group

29. Oxypoda operta Sjöberg* (NTR)

Irrasa Group

30. Oxypoda irrasa Mäklin

Inimica Group

31. Oxypoda yukonensis Klimaszewski \& Godin

Orbicollis Group

32. Oxypoda orbicollis Casey

33. Oxypoda frigida Bernhauer Grandipennis Group

34. Oxypoda grandipennis (Casey)

Amica Group

35. Oxypoda amica Casey (NTR)

\section{Phloeopora Erichson}

36. Phloeopora arctica Lohse

\section{Brachyusa Mulsant and Rey}

37. Brachyusa helenae (Casey) (NTR)

\section{Gnypeta Thomson}

Selmani Group

38. Gnypeta ashei Klimaszewski 
39. Gnypeta brincki Palm

40. Gnypeta sellmani Brundin**

Caerulea Group

41. Gnypeta caerulea** (C.R. Sahlberg)

IV. Tribe Hypocyphtini

\section{Cypha Leach}

42. Cypha inexpectata Klimaszewski \& Godin

V. Tribe Myllaenini Ganglbauer

\section{Myllaena Erichson}

Insomnis Group

43. Myllaena insomnis Casey

VI. Tribe Homalotini Heer

\section{Gyrophaena Mannerheim}

Nana Group

44. Gyrophaena nana (Paykull)**

45. Gyrophaena neonana Seevers

Keeni Group

46. Gyrophaena keeni Casey

Pulchella Group

47. Gyrophaena criddlei Casey (NTR) [tentative]

\section{Silusa Erichson}

48. Silusa californica (Bernhauer)

VII. Tribe Placusini Mulsant and Rey

\section{Placusa Erichson}

49. Placusa tacomae Casey

50. Placusa vaga Casey

VIII. Tribe Athetini Casey

\section{Acrotona Thomson}

51. Acrotona onthophila Lohse

52. Acrotona horwoodae Klimaszewski \& Godin, sp. n.

Mocyta Mulsant and Rey

53. Mocyta breviuscula (Mäklin)

54. Mocyta fungi (Gravenhorst)*

\section{Strigota Casey}

55. Strigota ambigua (Erichson) (NTR)

\section{Amischa Thomson}

56. Amischa praelonga (Casey) (NCR, NTR)

57. Amischa tersa Casey [tentative] 


\section{Atheta Thomson}

Subgenus Atheta Thomson

58. Atheta (s. str.) graminicola (Gravenhorst)**

59. Atheta (s. str.) martini Lohse

Subgenus Pseudota Casey

Klagesi Group

60. Atheta (Pseudota) klagesi Bernhauer

Subgenus Oreostiba Ganglbauer

61. Atheta (Oreostiba) sparreschneideri Munster**

Subgenus Alaobia Thomson

62. Atheta (Alaobia) ventricosa Bernhauer

Subgenus Bessobia Thomson

63. Atheta (Bessobia) cryptica (Lohse)

Subgenus Dimetrota Mulsant and Rey

Altaica Group

64. Atheta (Dimetrota) altaica Bernhauer **

65. Atheta (Dimetrota) nearctica (Lohse)

Prudhoensis Group

66. Atheta (Dimetrota) prudhoensis (Lohse)

67. Atheta (Dimetrota) burwelli (Lohse)

68. Atheta (Dimetrota) terranovae Klimaszewski \& Langor (NTR)

69. Atheta (Dimetrota) caribou (Lohse)

70. Atheta (Dimetrota) strigosula Casey

71. Atheta (Dimetrota) pseudometlakatlana Klimaszewski \& Godin

Modesta Group

72. Atheta (Dimetrota) pseudocrenuliventris Klimaszewski

Campbelli Group

73. Atheta (Dimetrota) smetanai (Lohse)

74. Atheta (Dimetrota) campbelli (Lohse)

Fanatica Group

75. Atheta (Dimetrota) fanatica Casey (NTR)

76. Atheta (Dimetrota) munsteri Bernhauer**

Cadeti Group

77. Atheta (Dimetrota) cadeti Klimaszewski and Godin

Subgenus Rhagocneme Munster

78. Atheta (Rhagocneme) subsinuata (Erichson)*

Subgenus Datomicra Mulsant and Rey

79. Atheta (Datomicra) dadopora Thomson* or **

80. Atheta (Datomicra) whitehorsensis Klimaszewski \& Godin, sp. n.

Subgenus Microdota Mulsant and Rey

81. Atheta (Microdota) platonoffi Brundin** (NTR)

82. Atheta (Microdota) pratensis (Mäklin) (NTR)

83. Atheta (Microdota) microelytrata Klimaszewski \& Godin, sp. n. 
84. Atheta (Microdota) riparia Klimaszewski \& Godin, sp. n.

SUBGENUS UNCERTAIN

85. Atheta brunswickensis Klimaszewski

86. Atheta capsularis Klimaszewski

87. Atheta remulsa Casey

\section{Dinaraea Thomson}

88. Dinaraea angustula (Gyllenhal)* (NTR)

89. Dinaraea planaris (Mäklin)

\section{Dochmonota Thomson}

90. Dochmonota rudiventris (Eppelsheim)* or **

\section{Hydrosmecta Thomson}

91. Hydrosmecta pseudodiosica Lohse

\section{Earota Mulsant and Rey}

92. Earota dentata (Bernhauer)

\section{Emmelostiba Pace}

93. Emmelostiba microptera (Lohse)

\section{Liogluta Thomson}

94. Liogluta aloconotoides Lohse

95. Liogluta granulosa Lohse

96. Liogluta trapezicollis Lohse

97. Liogluta nigropolita (Bernhauer)

\section{Lypoglossa Fenyes}

98. Lypoglossa angularis (Mäklin)

99. Lypoglossa franclemonti Hoebeke (NTR)

\section{Philhygra Mulsant and Rey}

100. Philhygra pseudopolaris Klimaszewski and Langor [listed as P. polaris

(Bernhauer) by Lohse et al. 1990]

101. Philhygra botanicarum (Muona)**

102. Philhygra pseudolarsoni Klimaszewski \& Godin, sp. n.

103. Philhygra sinuipennis Klimaszewski \& Langor (NTR)

104. Philhygra malleoides Lohse

105. Philhygra leechi Lohse (NTR)

106. Philhygra ripicoloides Lohse

107. Philhygra pseudoboreostiba Lohse

108. Philhygra juni Lohse

109. Philhygra clemens (Casey) (NTR)

110. Philhygra terrestris Klimaszewski \& Godin, sp. n.

111. Philhygra jarmilae Klimaszewski \& Langor (NTR)

\section{Boreophilia Benick}

112. Boreophilia islandica (Kraatz)**

113. Boreophilia nearctica Lohse

114. Boreophilia blatchleyi (Bernhauer \& Scheerpeltz)

115. Boreophilia venti (Lohse) 
116. Boreophilia nomensis (Casey) [Lohse et al. 1990 described this species as B. caseyiana Lohse, which was synonymized by Gusarov 2003]

117. Boreophilia caseyi Lohse

118. Boreophilia insecuta (Eppelsheim)**

119. Boreophilia gelida (J. Sahlberg)**

120. Boreophilia herschelensis Klimaszewski \& Godin, sp. n.

121. Boreophilia davidgei Klimaszewski \& Godin, sp. n.

\section{Boreostiba Lohse}

122. Boreostiba frigida (J. Sahlberg) ${ }^{* *}$ [= sibirica sensu Lohse in Lohse et al. 1990]

123. Boreostiba sibirica (Mäklin)**

124. Boreostiba parvipennis (Bernhauer)

125. Boreostiba lagunae Lohse

\section{Systematic account of new records and new species of Aleocharinae from the Yu-} kon territory

\section{Tribe Oxypodini Thomson}

\section{Calodera parviceps (Casey)}

http://species-id.net/wiki/Calodera_parviceps

Figs 1-10 in Assing 2008

\section{Distribution.}

\begin{tabular}{l|l}
\hline Origin & Nearctic \\
\hline Nearctic distribution & Canada: NS, NB, ON, YT; USA: RI \\
\hline YT distribution & YUKON (NTR): Whitehorse, Paddy's Pond, 60.7067, -135.0917, 6.V.2007, \\
& $\begin{array}{l}649 \mathrm{~m}, \text { litter sifting, mixed aspen and white spruce forest, B. Godin (ECW, } \\
\text { LFC) 2 females }\end{array}$ \\
\hline References & Casey 1894, Assing 2002, 2008 \\
\hline
\end{tabular}

\section{Gnathusa eva Fenyes}

http://species-id.net/wiki/Gnathusa_eva

Figs 1, 13, 14

\section{Distribution.}

\begin{tabular}{l|l}
\hline Origin & Nearctic \\
\hline Nearctic distribution & Canada (NTR): BC, YT; USA: CA \\
\hline YT distribution & YUKON: Whitehorse, Granger subdivision, coniferous woodchip pile, 60.7097, \\
& $\begin{array}{l}\text {-135.0996, 2.IX.2007, 661 m, pitfall trap, B. Godin (LFC) 1 male; same data } \\
\text { except: 3.V.2008 (LFC, ECW) 4 males, 2 females }\end{array}$ \\
\hline References & Fenyes 1910, 1920, Moore and Legner 1975, Majka and Klimaszewski 2008a \\
\hline
\end{tabular}



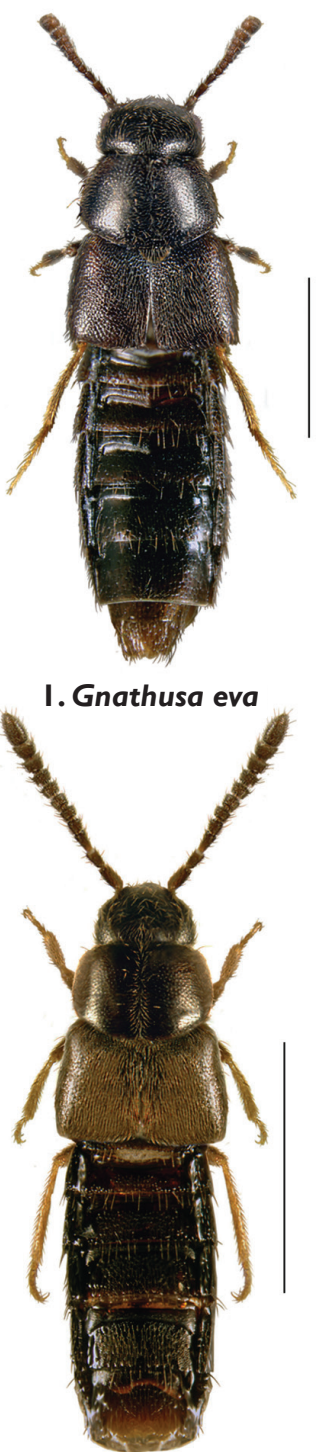

\section{Acronota horwoodae}

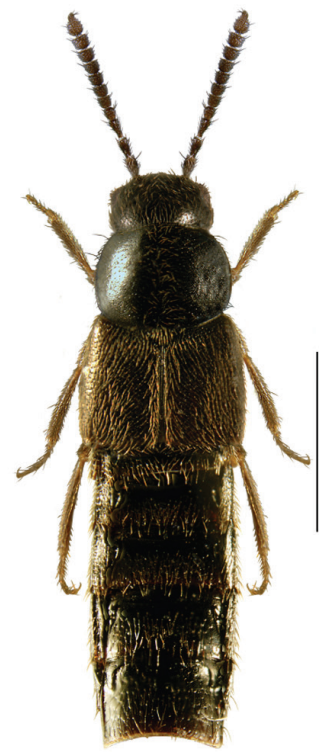

2. Gnathusa tenuicornis

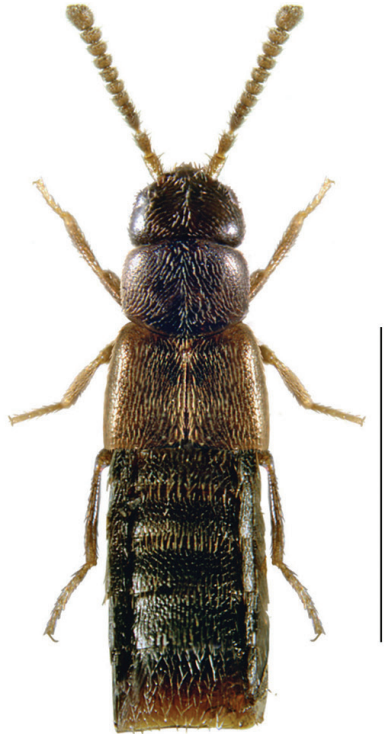

5. Amischa preolanga

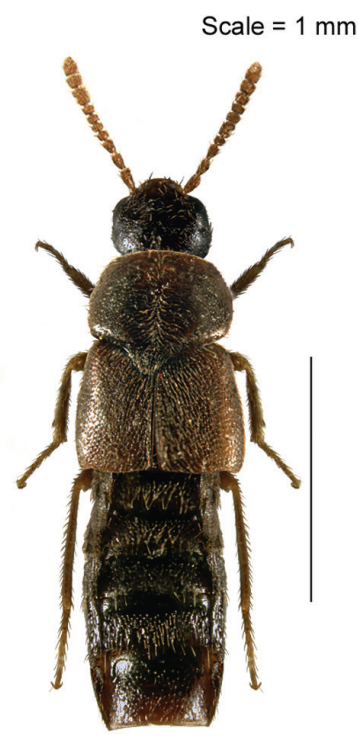

3. Ocyusa yukonensis

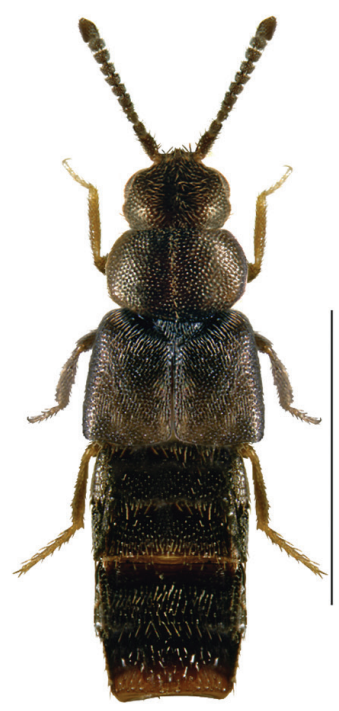

6. Atheta whitehorsensis

Figures I-6. Body images in dorsal view: I Gnathusa eva Fenyes $\mathbf{2}$ Gnathusa tenuicornis Fenyes $\mathbf{3}$ Ocyusa yukonensis Klimaszewski \& Godin, sp. n. 4 Acrotona horwoodae Klimaszewski \& Godin, sp. n. 5 Amischa praelonga (Casey) 6 Atheta (Datomicra) whitehorsensis Klimaszewski \& Godin, sp. n. 


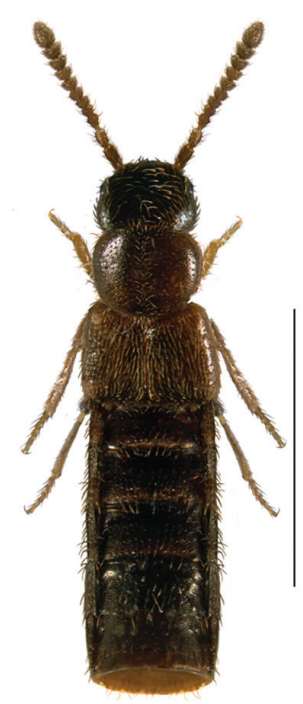

\section{Atheta microelytrata}

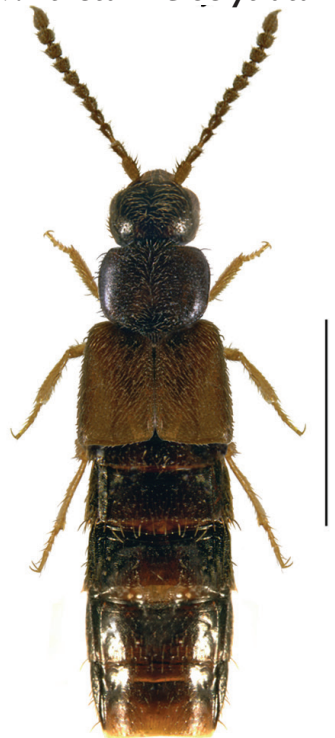

10. Philhygra terrestris

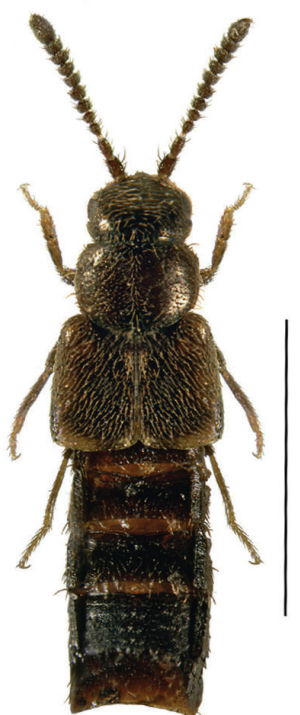

8. Atheta riparia

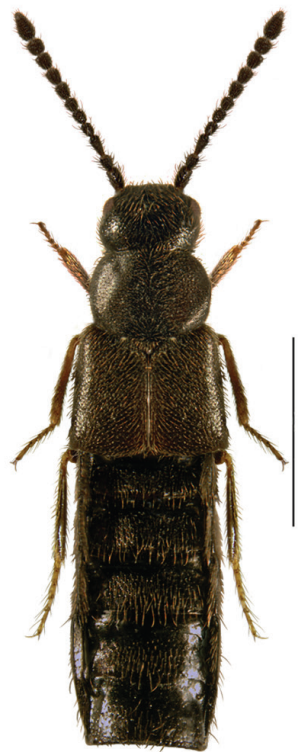

I I. Boreophilia herschelensis

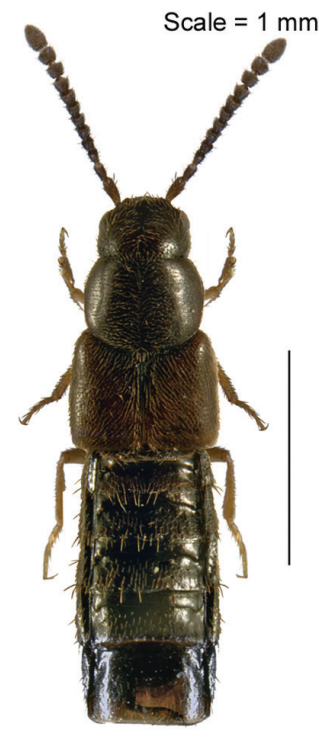

9. Philhygra pseudolarsoni

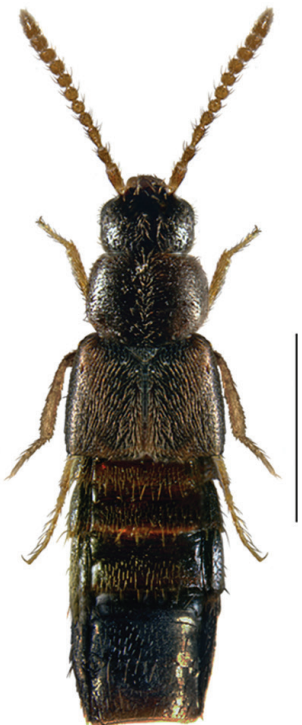

12. Boreophilia davidgei

Figures 7-12. Body images in dorsal view: 7 Atheta (Microdota) microelytrata Klimaszewski \& Godin, sp. n. 8 Atheta (Microdota) riparia Klimaszewski \& Godin, sp. n. 9 Philhygra pseudolarsoni Klimaszewski \& Godin, sp. n. 10 Philhygra terrestris Klimaszewski \& Godin, sp. n. II Boreophilia herschelensis Klimaszewski and Godin, sp. n. 12 Boreophilia davidgei Klimaszewski \& Godin, sp. n. 


\section{Gnathusa tenuicornis Fenyes}

http://species-id.net/wiki/Gnathusa_tenuicornis

Figs 2, 15

\section{Distribution.}

\begin{tabular}{l|l}
\hline Origin & Nearctic \\
\hline Nearctic distribution & Canada: YT, BC; USA: AK, CA \\
\hline YT distribution & $\begin{array}{l}\text { YUKON (NTR): Whitehorse, Paddy's Pond, 60.7067, -135.0917, 6.V.2007, 649 } \\
\text { m, litter sifting, mixed aspen and white spruce forest, B. Godin (ECW) 1 male }\end{array}$ \\
\hline References & $\begin{array}{l}\text { Fenyes 1921, Campbell and Davies 1991, Gouix and Klimaszewski 2007, } \\
\text { Moore and Legner 1975, Klimaszewski and Winchester 2002 }\end{array}$ \\
\hline
\end{tabular}

\section{Ocyusa yukonensis Klimaszewski \& Godin, sp. n.}

urn:lsid:zoobank.org:act:CAF7FE71-43FD-4C09-9B9C-FE58D3D72F29

http://species-id.net/wiki/Ocyusa_yukonensis

Figs 3, 16, 32, 33

Holotype (male). Canada, Yukon, EMAN Plot (Ecological Monitoring and Assessment Network), mature white spruce and feathermoss forest, 60.5963, -134.9522, 8.VII.2003, $738 \mathrm{~m}$, yellow pitfall trap (LMKM31Y), (LFC).

Paratype. Yukon, EMAN Plot, 60.5963, -134.9522, 24.VII.2003, 738 m, black pitfall trap (LMKM31B), (ECW) 1 male.

Etymology. Yukonensis - a Latin adjective derived from the Yukon Territory, Canada.

Diagnosis. Body small, subparallel, robust, uniformly dark brown, almost black; length $2.8-3.0 \mathrm{~mm}$; head round in outline and almost as wide as pronotum; antennae with article 4 subquadrate, 5-10 moderately transverse, increasingly wider apicad; pronotum transverse, angular posteriad and slightly narrower than maximum width of elytra; abdomen subparallel, at base as wide as elytra (Fig. 3). MALE: male tergite 8 widely truncate apically (Fig. 32); sternite 8 slightly produced at apex (Fig. 33); median lobe of aedeagus as illustrated (Fig. 16). FEMALE: unknown.

Distribution. This native Nearctic species is known only from the type locality in the Yukon.

Bionomics. Two adults were collected in July.

\section{Oxypoda canadensis Klimaszewski}

http://species-id.net/wiki/Oxypoda_canadensis

Figs 5, 41, 80-82, 171, 203, 204, 209, 210, in Klimaszewski et al. 2006

\section{Distribution.}

\begin{tabular}{l|l}
\hline Origin & Nearctic \\
\hline Nearctic distribution & Canada: NL, QC, ON, MB, AB, YT, NT; USA: AK, NH \\
\hline
\end{tabular}




\begin{tabular}{l|l}
\hline YT distribution & YUKON (NTR): Whitehorse, Paddy's Pond, 60.7067, -135.0917, 6.V.2007, 649 m, \\
& litter sifting, mixed aspen and white spruce forest, B. Godin (ECW) 1 male, 1 female; \\
& Watson Lake - Watson Creek, 60.1272, -128.805, 7.VII.2008, 697 m, deciduous \\
& debris soil sifting, B. Godin (ECW) 1 male, 2 females; Contact Creek, 65 km E \\
& Watson Lake; 59.9995, -127.7241,8.VI.2008, 621 m, litter sifting, creek bank, B. \\
& Godin (ECW) 1 male; Upper Liard, Albert Creek, 60.0522, -128.928, 8.VII.2008, \\
& 619 m, deciduous forest soil sifting, B. Godin (ECW, LFC) 3 males, 4 females \\
\hline References & Klimaszewski et al. 2006, Gouix and Klimaszewski 2007, Klimaszewski et al. 2011 \\
\hline
\end{tabular}

\section{Oxypoda operta Sjöberg* or **}

http://species-id.net/wiki/Oxypoda_operta

Figs 16, 52, 104, 105, 181, 245, 246, 249, 250, in Klimaszewski et al. 2006

\section{Distribution.}

\begin{tabular}{l|l}
\hline Origin & Holarctic or Palaearctic \\
\hline Nearctic distribution & Canada: NL, NS, QC, ON, AB, YT; USA: NH \\
\hline YT distribution & $\begin{array}{l}\text { YUKON (NTR): Watson Lake - Watson Creek, 60.1272, -128.805, 4.VI.2008, } \\
697 \text { m, deciduous debris, soil sifting, B. Godin (ECW) 1 male, 1 female }\end{array}$ \\
\hline References & $\begin{array}{l}\text { Smetana 2004, Klimaszewski et al. 2006, Gouix and Klimaszewski 2007, Majka } \\
\text { and Klimaszewski 2010, Klimaszewski et al. 2011 }\end{array}$ \\
\hline
\end{tabular}

\section{Brachyusa helenae (Casey)}

http://species-id.net/wiki/Brachyusa_helenae

Figs 48, 49, 222a-c, in Klimaszewski et al. 2011

\section{Distribution.}

\begin{tabular}{l|l}
\hline Origin & Nearctic \\
\hline Distribution & Canada: NL, YT, NT; USA: AK, MT \\
\hline YT distribution & $\begin{array}{l}\text { YUKON (NTR): Nisutlin Wildlife Area, 60.2317, -132.5632, 17.IX.2007, 679 m, } \\
\text { pitfall - Willow stand \#2 (ECW, LFC) 2 females }\end{array}$ \\
\hline References & $\begin{array}{l}\text { Casey 1911, Campbell and Davies 1991, Gouix and Klimaszewski 2007, Klimaszewski } \\
\text { et al. 2011 }\end{array}$ \\
\hline
\end{tabular}

\section{Tribe Homalotini Heer}

\section{Gyrophaena criddlei Casey}

http://species-id.net/wiki/Gyrophaena_criddlei

Figs 16, 107-110, in Klimaszewski et al. 2009

\section{Distribution.}

\begin{tabular}{l|l}
\hline Origin & Nearctic \\
\hline Distribution & Canada: NL, NB, MB, YT \\
\hline
\end{tabular}




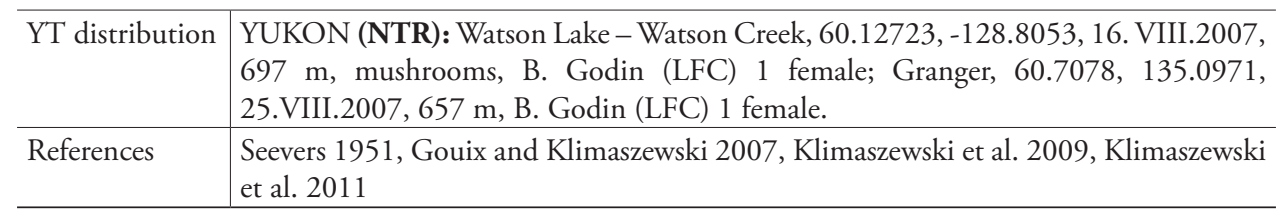

Comments. The two females are tentatively identified as G. criddlei but a male is needed for positive confirmation of this species in the Yukon Territory.

\section{Tribe Athetini Casey}

\section{Acrotona horwoodae Klimaszewski \& Godin, sp. n.} urn:lsid:zoobank.org:act:D5CA8598-36E8-40B4-AEAD-20D013A6964E http://species-id.net/wiki/Acrotona_horwoodae Figs 4, 17, 18, 34-37

Holotype (male). Canada, Yukon, Whitehorse, Paddy's Pond, 60.7067, -135.0917, 27.V.2008, $649 \mathrm{~m}$, litter sifting, mixed aspen and white spruce forest, B. Godin (LFC).

Paratype (female). Same data as the holotype (ECW).

Etymology. This species name is dedicated to Denise Horwood, wife of the second author, who assisted him in numerous aleocharine sample collections.

Diagnosis. Body narrowly oval, moderately convex, uniformly black, punctation on forebody fine, dense and not asperate, microsculpture fine but not pronounced; length $2.4 \mathrm{~mm}$; head narrower than pronotum, ratio of maximum width of head to maximum width of pronotum 0.7 ; antennal articles 7-10 slightly transverse; pronotum moderately transverse, ratio of maximum width to length 1.4 , about as wide as elytra; elytra at suture about as long as pronotum; abdomen slightly narrowed posteriad (Fig. 4). MALE: tergite 8 moderately elongate and truncate apically (Fig. 34); sternite 8 widely arcuate apically (Fig. 35); median lobe of aedeagus as illustrated (Fig. 17). FEMALE: tergite 8 moderately elongate and truncate apically, base not sinuate (Fig. 36); sternite 8 widely arcuate apically, base not sinuate (Fig. 37); spermatheca with capsule tulip-shaped and stem coiled posteriorly (Fig. 18).

Bionomics. The specimens were found by sifting forest litter in May.

Comments. The shape of the median lobe of the aedeagus and the spermatheca of A. horwoodae are different from all recorded species of Nearctic Acrotona, and they are generally similar to those of the Palaearctic species $A$. aterrima Gravenhorst, which is brown and has a much broader body. 


\section{Strigota ambigua (Erichson)}

http://species-id.net/wiki/Strigota_ambigua

Figs 88, 261a-c, in Klimaszewski et al. 2011

\section{Distribution.}

\begin{tabular}{l|l}
\hline Origin & Nearctic \\
\hline Distribution & $\begin{array}{l}\text { Canada: NL, NS, PE, YT; USA: CA, CO, CT, IA, KS, MA, MO, NC, NJ, NV, NY, } \\
\text { TX }\end{array}$ \\
\hline YT distribution & $\begin{array}{l}\text { YUKON (NTR): Whitehorse, 60.7328, -135.0986 18.VI.2007, } 717 \text { m, hand } \\
\text { collected, parking lot asphalt, (ECW) 1 female }\end{array}$ \\
\hline References & $\begin{array}{l}\text { Bernhauer 1907, Gusarov 2003, Gouix and Klimaszewski 2007, Majka et al. 2008b, } \\
\text { Majka and Klimaszewski 2010, Klimaszewski et al. 2011 }\end{array}$ \\
\hline
\end{tabular}

\section{Amischa praelonga (Casey)}

http://species-id.net/wiki/Amischa_praelonga

Figs 5, 19, 38, 39

\section{Distribution.}

\begin{tabular}{l|l}
\hline Origin & Nearctic \\
\hline Distribution & Canada (NTR): YT; USA: WY \\
\hline YT distribution & YUKON (NTR): Whitehorse, McIntyre Creek, 60.7398, -135.1462, 25.IV.2007, \\
& $\begin{array}{l}\text { 744 m, litter sifting, willow stand by creek bank, B. Godin (ECW, LFC) 2 females; EP } \\
\text { Impact, south, 60.7336, -135.0946,19.VII.2001, 695 m, pitfall trap, disturbed land, } \\
\text { grasses, B. Godin (ECW, LFC) 3 females }\end{array}$ \\
\hline References & Casey 1894 \\
\hline
\end{tabular}

Comments. Two additional Amischa morphotypes were recognized in the Yukon material on the basis of external body characters and the shape of the spermatheca. They are not included in this account because they are difficult to associate with any of the recorded species. The first morphospecies is represented by three narrowly elongate bicoloured specimens with the head and 4-5 basal abdominal tergites almost black, with the pronotum brown and the appendages and posterior of the elytra light brown, and with the spermathecal capsule moderately elongate with a moderately long apical invagination. The second morphospecies is represented by three specimens, which are broader, with the body uniformly dark brown to almost black, and the spermathecal capsule broader and shorter apically and with a longer apical invagination. Both groups have the apex of tergite 8 deeply notched. We need more specimens and representatives of both sexes to establish the status of these morphotypes. 


\section{Atheta (Dimetrota) terranovae Klimaszewski \& Langor}

http://species-id.net/wiki/Atheta_terranovae

Figs 107, 280a-c, 407a-d, in Klimaszewski et al. 2011

\section{Distribution.}

\begin{tabular}{l|l}
\hline Origin & Nearctic \\
\hline Distribution & Canada: NL, YT \\
\hline YT distribution & YUKON (NTR): Whitehorse, Granger, 60.7078, -135.0971, 1.VIII.2007, 657 \\
& m, mushrooms, B. Godin (ECW) 2 females; same data except: 60.7366, 135.097, \\
& 15.VIII.2008, 743 m, pitfall trap, ski trail, birch stand, B.Godin (ECW) 1 male; \\
& $\begin{array}{l}\text { EMAN Plot, Fireweed Dr., 60.6014,-134.9387, 8.VIII.2006, 772 m, pitfall trap, } \\
\text { mixed pine and willow forest (ECW) 1 male; same data except: 23.VII.2006 (ECW) } \\
1 \text { female; EMAN Plot, Cadet Camp, 60.5951, -134.9499, 23.VIII.2006, 760 m, } \\
\text { pitfall trap, mature white spruce and feathermoss forest, (ECW) 1 female }\end{array}$ \\
\hline References & Klimaszewski et al. 2011 \\
\hline
\end{tabular}

\section{Atheta (Dimetrota) fanatica Casey}

http://species-id.net/wiki/Atheta_fanatica

Figs 134, 307a-c, in Klimaszewski et al. 2011

\section{Distribution.}

\begin{tabular}{l|l}
\hline Origin & Nearctic \\
\hline Distribution & Canada: NL, NS, NB, QC, YT, BC; USA: AK, NV \\
\hline YT distribution & YUKON (NTR): Whitehorse, Paddy's Pond, 60.7067, -135.0917, 20.V.2007, 649 \\
& m, litter sifting, B. Godin (ECW) 1 male; Whitehorse, Granger, 60.7078, -135.0971, \\
& 5.VIII.2007, 657 m, soil sifting, B. Godin (ECW) 1 male; same data except: \\
& 27.IX.2008, compost (LFC) 1 male, 1 female \\
\hline References & $\begin{array}{l}\text { Campbell and Davies 1991, Casey 1910, 1911, Moore and Legner 1975, Majka et } \\
\text { al. 2006 [as irrita], Webster et al. } 2009 \text { [as irrita], Majka and Klimaszewski } 2010 \text { [as } \\
\text { irrita], Klimaszewski et al. 2011 }\end{array}$ \\
\hline
\end{tabular}

\section{Atheta (Datomicra) whitehorsensis Klimaszewski \& Godin, sp. n.} urn:lsid:zoobank.org:act:9ACD0F86-341A-4855-925A-51104BB8C8F4 http://species-id.net/wiki/Atheta_whitehorsensis Figs 6, 20, 21, 40-43

Holotype (male). Canada, Yukon, Whitehorse, Granger, 60.7078, -135.0971, 25.VIII.2007, 657 m, soil sifting, black spruce stand, AWT, B. Godin (LFC).

Paratype. Canada, Yukon, Whitehorse, Granger, 60.7078, -135.0971, 5.VIII.2007, 657 m, soil sifting, black spruce stand, AWT, B. Godin (ECW) 1 female.

Etymology. The specific name derives from the name of the type locality, which is Whitehorse, Yukon.

Diagnosis. Body narrowly oval, dark brown to black, with bases of antennae and legs rust-brown, surface matte, with asperate dense punctation on forebody and strong 
meshed microsculpture (Fig. 6); length 1.9-2.0 mm; head narrower than pronotum and elytra, with short postocular area, eyes large and slightly protruding; antennae slender, slightly incrassate apically, article 4 subquadrate, 5 slightly elongate and 6-10 slightly to strongly transverse; pronotum strongly transverse and broadest in the middle; elytra transverse, longer than pronotum; abdomen broadly arcuate laterally (Fig. 6). MALE: tergite 8 transverse and truncate apically (Fig. 40); sternite 8 widely rounded apically (Fig. 41); median lobe of aedeagus with venter of tubus straight and short, and apex sharply produced (Fig. 20). FEMALE: tergite and sternite 8 truncate apically (Figs 42, 43); spermatheca with pipe-shaped capsule and long stem hooked posteriorly (Fig. 21).

This species is similar externally to Atheta (Dimetrota) hampshirensis Bernhauer and Atheta (Datomicra) dadopora Thomson but differs in the shape of the spermatheca and median lobe of the aedeagus, and has a broader body than the latter species.

Distribution. This native Nearctic species is known only from the type locality in the Yukon Territory.

Bionomics. Adults were captured by sifting soil in a black spruce stand.

\section{Atheta (Microdota) platonoffi Brundin**}

http://species-id.net/wiki/Atheta_platonoffi

Figs 127, 300a-c, 423, in Klimaszewski et al. 2011

\section{Distribution.}

\begin{tabular}{l|l}
\hline Origin & Holarctic \\
\hline Distribution & Canada: NL, NS, NB, ON, AB, BC, YT; USA: AK \\
\hline YT distribution & $\begin{array}{l}\text { YUKON (NTR): Whitehorse, Granger, 60.7078, -135.0971, 25.VIII.2007, 657 m, } \\
\text { soil sifting, black spruce stand, B. Godin (ECW, LFC) 3 males, 2 females; same data } \\
\text { except: 1.VIII.2008, mushrooms (ECW, LFC) 3 males; 16.VIII.2007, mushrooms } \\
\text { (ECW) 1 female; Upper Liard, Albert Creek, 60.0522, -128.928, 8.VII.2007, 699 } \\
\text { m, deciduous debris, soil sifting, B. Godin (ECW) 1 female }\end{array}$ \\
\hline References & $\begin{array}{l}\text { Klimaszewski et al. 2005, Gouix and Klimaszewski 2007, Majka et al. 2008b, 2010, } \\
\text { Klimaszewski et al. 2011 }\end{array}$ \\
\hline
\end{tabular}

Atheta (Microdota) pratensis (Mäklin)

http://species-id.net/wiki/Atheta_pratensis

Figs 128, 301a-c, 428, in Klimaszewski et al. 2011

\section{Distribution.}

\begin{tabular}{l|l}
\hline Origin & Nearctic \\
\hline Distribution & Canada: NL, YT; USA: AK \\
\hline YT distribution & $\begin{array}{l}\text { YUKON (NTR): Tagish, Tagish Lake; 60.2658, -134.2873, 20.VIII.2007, 654 m, } \\
\text { mushroom, B. Godin (ECW) 1 male }\end{array}$ \\
\hline References & Mäklin 1853, Klimaszewski et al. 2011 \\
\hline
\end{tabular}




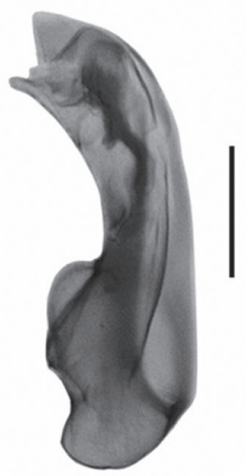

13. Gnathusa eva

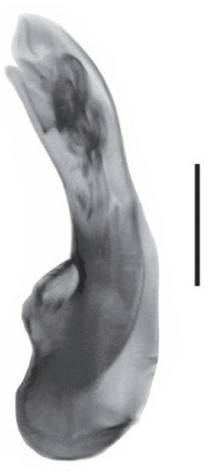

16. Ocyusa yukonensis

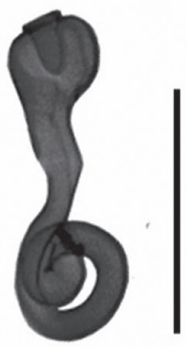

19. Amischa praelonga

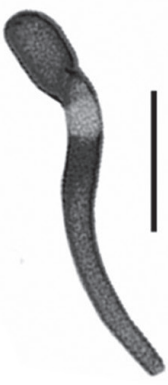

14

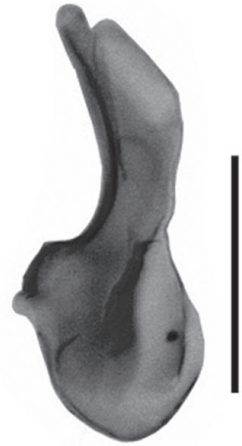

17. Acronota horwoodae

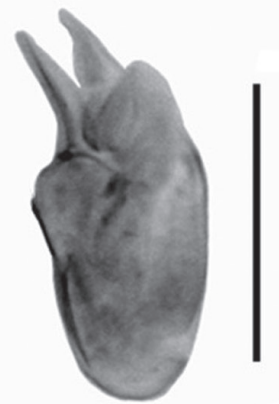

20. Atheta whitehorsesis

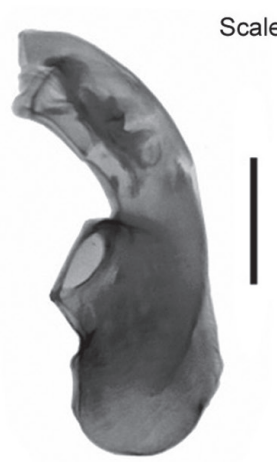

I5. Gnathusa tenuicornis

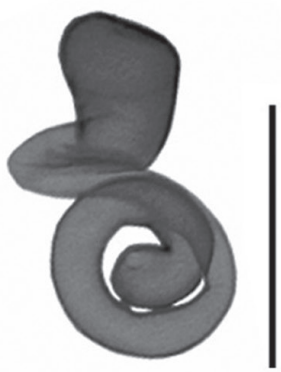

18

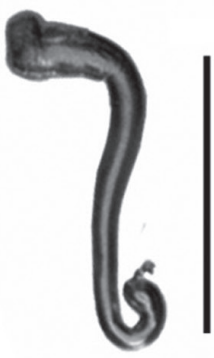

21

Figures | 3-2 I. Median lobe of aedeagus and spermatheca in lateral view of Gnathusa eva Fenyes I 3, I 4 Gnathusa tenuicornis Fenyes 15 Ocyusa yukonensis Klimaszewski \& Godin, sp. n. 16 Acrotona horwoodae Klimaszewski \& Godin, sp. n. I7, 18 Amischa praelonga (Casey) 19 Atheta (Datomicra) whitehorsensis Klimaszewski \& Godin, sp. n. 20, 2 I. 


\section{Atheta (Microdota) microelytrata Klimaszewski \& Godin, sp. n.} urn:Isid:zoobank.org:act:A75DCD78-E696-4AE7-8E8C-ACAF8F3B3F7E http://species-id.net/wiki/Atheta_microelytrata Figs 7, 22, 23, 44-47

Holotype (male). Canada, Yukon, Whitehorse, Takhini, hotsprings, 60.8769, -135.3596, 30.IV.2009, $716 \mathrm{~m}$, aspen litter - soil sifting, B. Godin (LFC).

Paratypes. Canada, Yukon, Whitehorse, Takhini, hotsprings, 60.8769, -135.3596, 19.IX.2009, 716 m, alder/willow litter, soil sifting, B. Godin (ECW) 2 males; same data except: 3.V.2009 (ECW, LFC) 2 females.

Etymology. The specific name derives from the word micro, meaning small, and elytra, in allusion to the small and short elytra of this species.

Diagnosis. Body narrowly subparallel; dark brown, with bases of antennae and legs rust-brown; strongly glossy, with fine and moderately dense punctation on forebody and strong, meshed microsculpture (Fig. 7); head as wide as pronotum and elytra, with long postocular area, eyes moderately small and slightly protruding; antennae slender, slightly incrassate apicad, articles $4-5$ subquadrate and 6-10 slightly to strongly transverse; pronotum narrower at base and broadening apicad; elytra transverse, shorter than pronotum; abdomen widest subapically; length $1.9-2.0 \mathrm{~mm}$ (Fig. 7). MALE: tergite 8 truncate apically and with crenulation scarcely visible (Fig. 44); sternite 8 widely rounded apically (Fig. 45); median lobe of aedeagus with apex narrow and ventrally produced, athetine bridge well developed (Fig. 22). FEMALE: tergite 8 truncate apically (Fig. 46); sternite 8 truncate and slightly emarginate medially (Fig. 47); spermatheca with pipe-shaped capsule and long, posteriorly-coiled stem (Fig. 23).

This species bears some superficial external similarity to Geostiba and Emmelostiba but has typical Atheta-like genitalia.

Distribution. This native Nearctic species is known only from the type locality in the Yukon Territory.

Bionomics. Adults were found in aspen, alder and willow litter in March, May and September.

\section{Atheta (Microdota) riparia Klimaszewski \& Godin, sp. n.}

urn:lsid:zoobank.org:act:BC82DFB4-F60B-4758-9860-BC23B2F3D6DC http://species-id.net/wiki/Atheta_riparia

Figs 8, 24, 25, 48-51

Holotype (male). Canada, Yukon, Whitehorse, Paddy's Pond, 60.7067, -135.0917, 16.IX.2007, 649 m, litter sifting, mixed aspen and white spruce forest, B. Godin (LFC).

Paratype. Same data as the holotype (ECW) 1 male.

Non-type. Canada, Yukon, Watson Lake, Watson Creek, 60.12723, -128.8053, 16.VIII.2007, 697 m, mushrooms, B. Godin (LFC) 1 female. 
Etymology. The name of this species derives from the Latin adjective riparius, $-a$, $-u m$, in allusion to the wet litter where the types were found.

Diagnosis. Body small and narrow, subparallel; black, with tarsi reddish-brown; moderately glossy, with fine, dense punctation and meshed microsculpture on forebody (Fig. 8); head approximately as wide as pronotum, depressed medially, eyes slightly protruding; antennae slender, slightly incrassate apicad, articles 4-10 slightly to strongly transverse; pronotum emarginate laterally; elytra broader and longer at suture than pronotum; head, pronotum and base of abdomen of the same width; sides of abdomen subparallel; length 1.9-2.0 mm (Fig. 8). MALE: tergite 8 truncate apically and with smooth margin (Fig. 48); sternite 8 widely rounded apically (Fig. 49); median lobe of aedeagus with apex narrow and ventrally produced (Fig. 24). FEMALE (non-paratype): tergite 8 truncate apically (Fig. 50); sternite 8 broadly rounded apically (Fig. 51); spermatheca slightly distorted but with club-shaped capsule and posteriorly-twisted stem (Fig. 25).

This species differs from other Nearctic Microdota by the combination of body shape, strongly punctate surface and the shape of the median lobe of the aedeagus and spermatheca.

Distribution. This native Nearctic species is known only from the Yukon Territory but it is probably more widely distributed in northern Canada.

Bionomics. The two males were captured in September in wet, organic litter and the female was found in mushrooms in mid-August.

\section{Dinaraea angustula (Gyllenhal)*}

http://species-id.net/wiki/Dinaraea_angustula

Figs 141, 314a-c, 442, in Klimaszewski et al. 2011

\section{Distribution.}

\begin{tabular}{l|l}
\hline Origin & Palaearctic \\
\hline Distribution & Canada: NL, NS, NB, PE, QC, ON, AB, YT; USA: CA, NY \\
\hline YT distribution & $\begin{array}{l}\text { YUKON (NTR): EMAN plot, Cadet Camp, 60.5951, -134.9499, 26.V.2006, 760 } \\
\text { m, pitfall trap, mature white spruce and feathermoss forest, B. Godin (LFC) 1 male }\end{array}$ \\
\hline References & $\begin{array}{l}\text { Moore and Legner 1975, Muona 1984, Smetana 2004, Klimaszewski et al. 2007, } \\
\text { Gouix and Klimaszewski 2007, Webster et al. 2009, Majka et al. 2008b, 2010, } \\
\text { Klimaszewski et al. 2011 }\end{array}$ \\
\hline
\end{tabular}

\section{Lypoglossa franclemonti Hoebeke}

http://species-id.net/wiki/Lypoglossa_franclemonti

Figs 154, 328a-c, 455, in Klimaszewski et al. 2011

\section{Distribution.}

\begin{tabular}{l|l}
\hline Origin & Nearctic \\
\hline Distribution & Canada: NL, NB, NS, QC, ON, MB, AB, YT, NT; USA: ME, NH, NY, VT \\
\hline YT distribution & YUKON (NTR): Upper Liard, Albert Creek, 60.0522, -128.9279, 3.VI.2007, 699 m, \\
& deciduous litter sifting, B. Godin (ECW, LFC) 4 males, 2 females; same data except: \\
& 4.VI.2007 (ECW, LFC) 1 male, 2 females, 7.VII.2008 (ECW, LFC) 2 males; Watson \\
& Lake, Watson Creek, 60.12723, -128.8053, 16.VIII.2007, 697 m (ECW) 1 male \\
\hline References & Hoebeke 1992, Gusarov 2004, Gouix and Klimaszewski 2007, Klimaszewski et al. 2011 \\
\hline
\end{tabular}




\section{Philhygra pseudolarsoni Klimaszewski \& Godin, sp. n. urn:lsid:zoobank.org:act:64A996FC-47AE-453A-A112-B57D0C0D950F http://species-id.net/wiki/Philhygra_pseudolarsoni}

Figs 9, 26, 52-55

Holotype (male). Canada, Yukon, Whitehorse, Paddy's Pond, 60.7067, -135.0917, 26.V.2007, $649 \mathrm{~m}$, litter sifting, mixed aspen and white spruce forest, B. Godin (LFC).

Paratypes. same label data as the holotype (ECW) 1 male; Watson Lake, Watson Creek, 60.1272, -128.8053, 4.VI.2007, 697 m, deciduous forest soil sifting, B. Godin (ECW) 1 male, 1 female.

Etymology. This species name derives from the specific name larsoni ( $P$. larsoni Klimaszewski and Langor), and the prefix pseudo (false) in relation to the similarity of the two species in external and, to a lesser degree, genitalic morphology.

Diagnosis. Body narrowly subparallel, uniformly black or black with legs and sutural part of elytra reddish-brown (Fig. 9); moderately glossy, with fine, dense punctation and meshed microsculpture on forebody; head round, distinctly narrower than pronotum, with eyes as long as postocular region of head; antennae slender with articles 4-5 elongate, 6-10 subquadrate to slightly transverse; pronotum slightly transverse and almost as wide as elytra; elytra at suture as long as or slightly longer than pronotum; length $2.9-3.0 \mathrm{~mm}$ (Fig. 9). MALE: tergite 8 widely arcuate apically (Fig. 52); sternite 8 elongate and rounded apically (Fig. 53); median lobe of aedeagus with apex triangularly produced in lateral view (Fig. 26).

Female. tergite 8 truncate apically (Fig. 54); sternite 8 produced medially (Fig. 55); pygidium with ventral structure weakly sclerotized.

Distribution. This species is known only from Whitehorse and Watson Lake in the Yukon Territory.

Bionomics. This species was collected in May and June from ground litter.

Comments. Philhygra pseudolarsoni is similar in both external morphology and genitalia to $P$. larsoni Klimaszewski and Langor. However, it may be distinguished from $P$. larsoni by the smaller and darker body, quadrate or transverse antennal articles $4-10$ and by the median lobe of the aedeagus with a more elongate apical part of the tubus in lateral view.

\section{Philhygra sinuipennis Klimaszewski \& Langor}

http://species-id.net/wiki/Philhygra_sinuipennis

Figs 161, 335a, b, 462a, b, in Klimaszewski et al. 2011

\section{Distribution.}

\begin{tabular}{l|l}
\hline Origin & Nearctic \\
\hline Distribution & Canada: NL, YT \\
\hline YT distribution & $\begin{array}{l}\text { YUKON (NTR): Watson Lake, Watson Creek, 60.1272, -128.8053, 4.VI.2007, 697 } \\
\text { m, deciduous litter sifting, B. Godin (ECW, LFC) 2 males }\end{array}$ \\
\hline References & Klimaszewski et al. 2011 \\
\hline
\end{tabular}




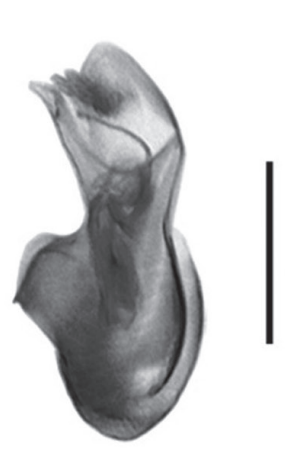

22. Atheta microelytrata

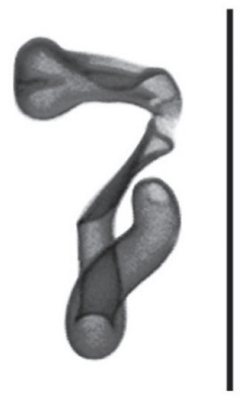

25

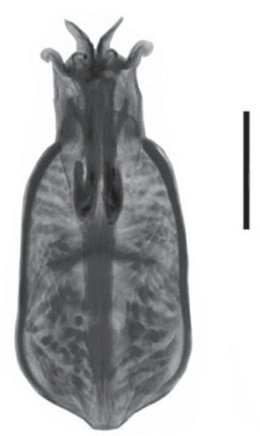

28. Boreophilia hershelensis

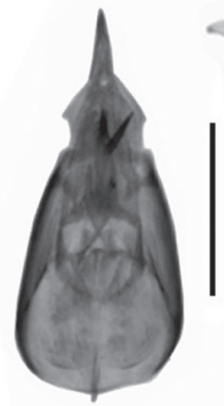

a

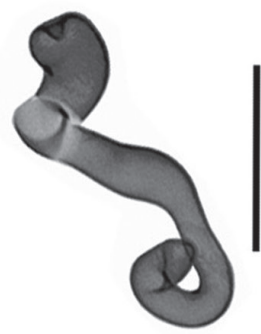

23

26. Phlihygra pseudolarsoni

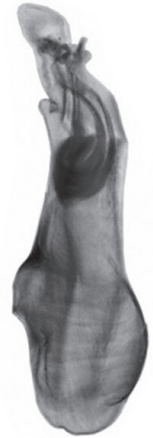

29

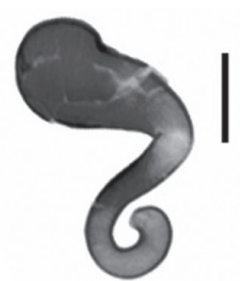

30

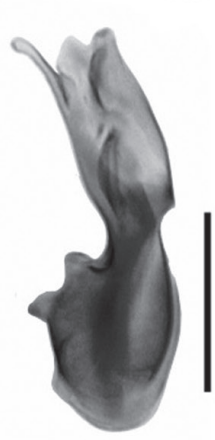

24. Atheta riparia

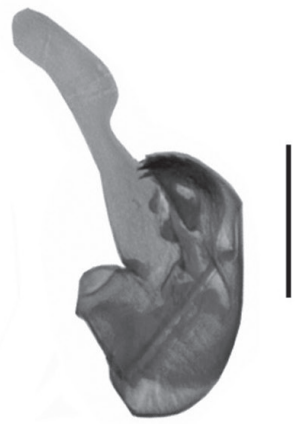

27. Philhygra terrestris

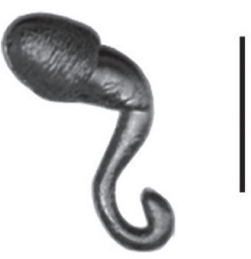

31. Boreophilia davidgei

Figures 22-3I. Median lobe of aedeagus and spermatheca (view as specified) of Atheta (Microdota) microelytrata Klimaszewski and Godin, sp. n. 22 lateral 23 lateral; Atheta (Microdota) riparia Klimaszewski \& Godin, sp. n. 24 lateral 25 lateral; Philhygra pseudolarsoni Klimaszewski \& Godin, sp. n. 26 lateral; Philhygra terrestris Klimaszewski \& Godin, sp. n. 27 lateral; Boreophilia herschelensis Klimaszewski \& Godin, sp. n. 28 dorsal 29 lateral 30 lateral; Boreophilia davidgei Klimaszewski \& Godin, sp. n. 3 I lateral. 


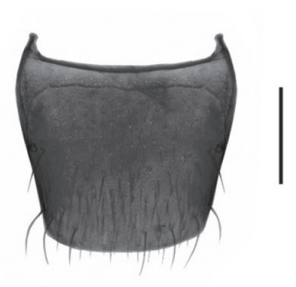

32. Ocyusa yukonensis

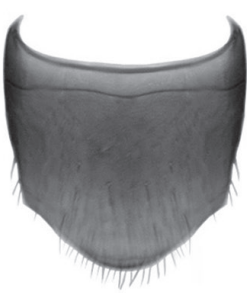

33
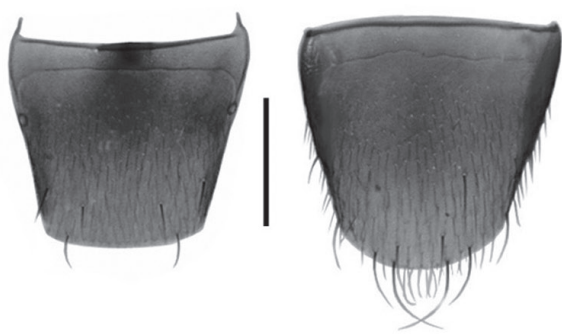

34. Acronota horwoodae

35

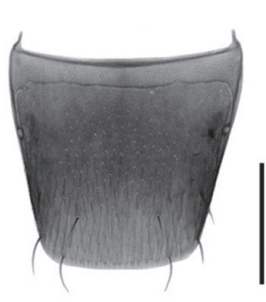

36

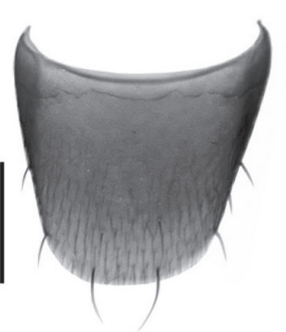

37

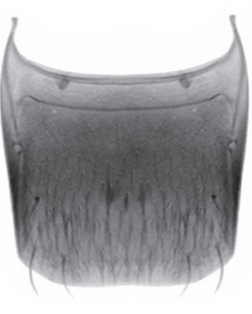

38. Amischa praelonga

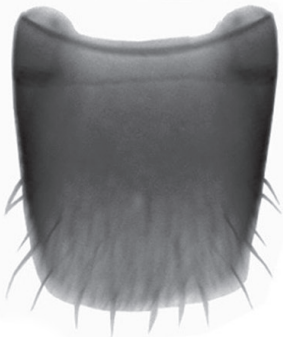

39
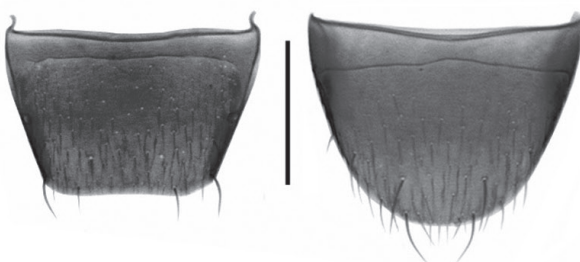

41

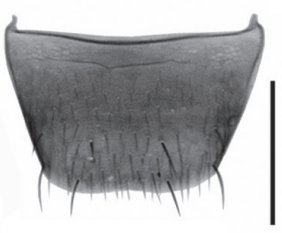

42

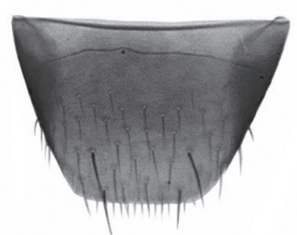

43

Figures 32-43. Male and female tergite and sternite 8: Ocyusa yukonensis Klimaszewski \& Godin, sp. n. 32, 33 male; Acrotona horwoodae Klimaszewski \& Godin, sp. n. 34, 35, male 36, 37, female Amischa praelonga (Casey) 38, 39 female; Atheta (Datomicra) whitehorsensis Klimaszewski \& Godin, sp. n. 40, 4 I male 42, 43 female. 

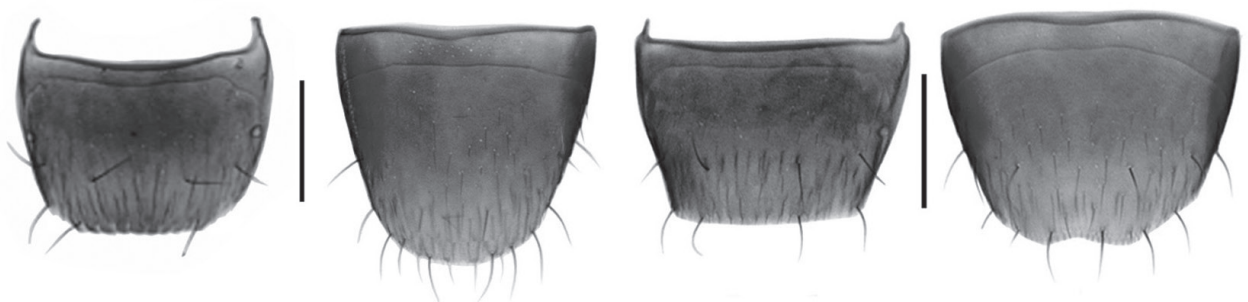

44. Atheta microelytrata

45

46

47
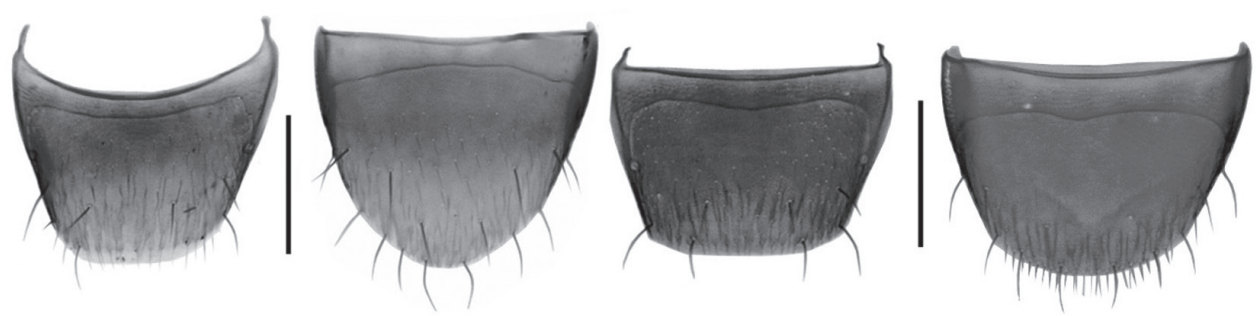

48. Atheta riparia

49

50

5 I

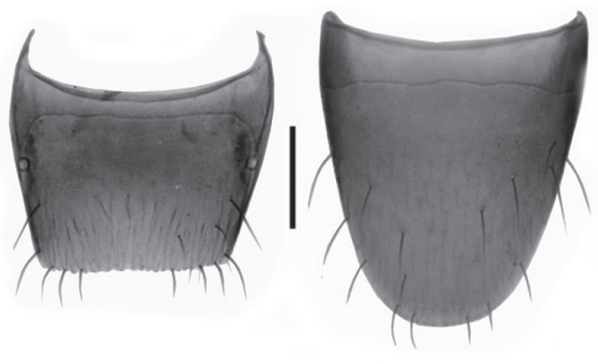

52. Philhygra pseudolarsoni

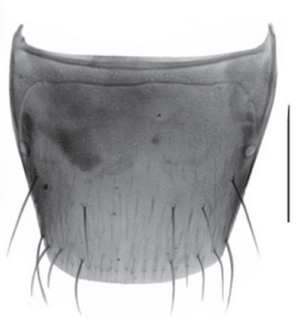

54

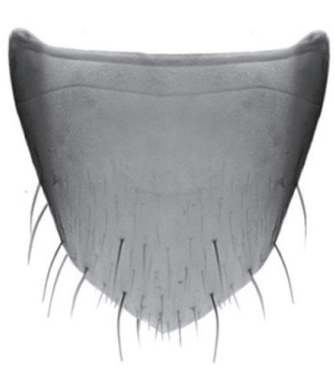

55

Figures 44-55. Male and female tergite and sternite 8: Atheta (Microdota) microelytrata Klimaszewski \& Godin, sp. n. 44, 45 male 46, 47 female; Atheta (Microdota) riparia Klimaszewski \& Godin, sp. n. 48, 49 male 50, 5 I female; Philhygra pseudolarsoni Klimaszewski \& Godin, sp. n. 52, 53, male 54, 55 female. 


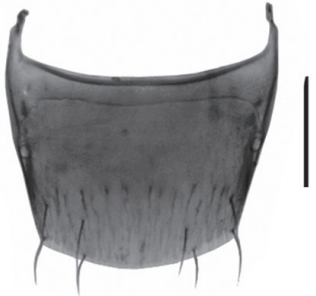

56. Philhygra terrestris

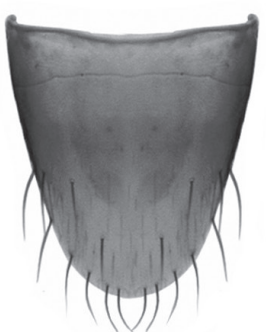

57

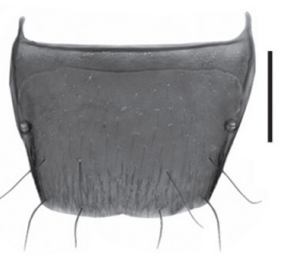

58. Boreophilia herschelensis

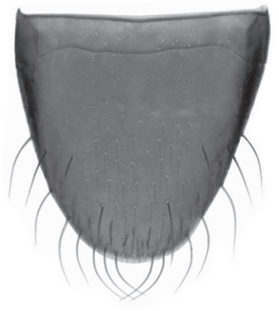

59
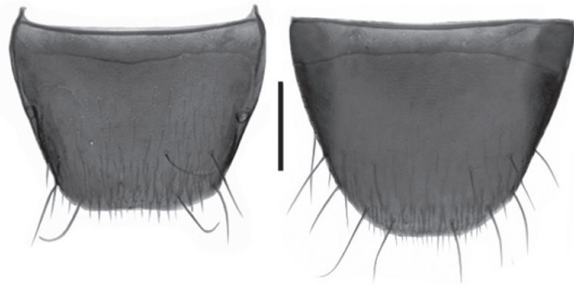

61

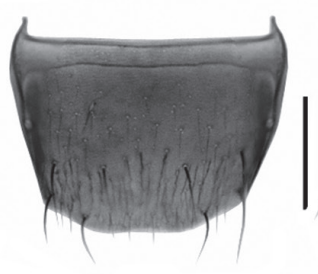

62. Boreophilia davidgei

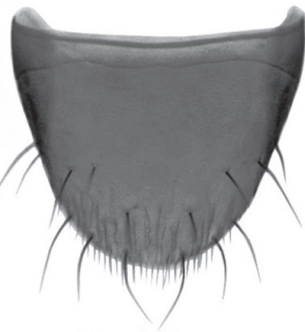

63

Figures 56-63. Male and female tergite and sternite 8: Philhygra terrestris Klimaszewski \& Godin, sp. n. 56, 57 male; Boreophilia herschelensis Klimaszewski \& Godin, sp. n. 58, 59 male 60, 6I female; Boreophilia davidgei Klimaszewski \& Godin, sp. n. 62, 63 female.

\section{Philhygra leechi Lohse}

http://species-id.net/wiki/Philhygra_leechi

Figs 118, 119, in Lohse et al. 1990

\section{Distribution.}

\begin{tabular}{l|l}
\hline Origin & Nearctic \\
\hline Distribution & Canada: MB, YT, NT \\
\hline YT distribution & $\begin{array}{l}\text { YUKON (NTR): Nisutlin Wildlife Area, 60.2317, -132.5632, 21.VIII.2007, 679 m, } \\
\text { pitfall - Willow stand \# 2, B. Godin (LFC) 1 male. }\end{array}$ \\
\hline References & Lohse et al. 1990, Gouix and Klimaszewski 2007 \\
\hline
\end{tabular}




\section{Philhygra terrestris Klimaszewski \& Godin, sp. n.}

urn:lsid:zoobank.org:act:246EBFF8-C0AE-43D6-98D9-C99289EE7B47

http://species-id.net/wiki/Philhygra_terrestris

Figs 10, 27, 56, 57

Holotype (male). Canada, Yukon, Whitehorse, Paddy's Pond, 60.7067, -135.0917, 26.V.2007, $649 \mathrm{~m}$, litter sifting, mixed forest (aspen and white spruce), B. Godin (LFC).

Etymology. This species name is an adjective that derives from the Latin word terra (ground, earth, soil).

Diagnosis. Body narrowly subparallel, head and abdomen black, pronotum and elytra brown, basal article of antenna and legs yellowish (Fig. 10); strongly glossy, with fine, dense punctation and meshed microsculpture on forebody; head round, distinctly narrower than pronotum with eyes as long as postocular region of head; antennae slender with articles $4-5$ elongate, 6-10 subquadrate; pronotum slightly transverse and almost as wide as elytra; elytra at suture slightly longer than pronotum; length 2.9-3.0 mm (Fig. 10). MALE: tergite 8 widely arcuate apically (Fig. 56); sternite 8 elongate and rounded apically (Fig. 57); aedeagus with apex of median lobe broadly produced and with tubus constricted basally in lateral view (Fig. 27).

Female. unknown.

Distribution. This species is known only from Whitehorse in the Yukon but it may be more widely distributed in the boreal zone of Canada and Alaska.

Bionomics. This species was collected in May from ground litter.

Comments. This species is unique in the shape of the median lobe of the aedeagus in lateral view.

\section{Philhygra jarmilae Klimaszewski \& Langor}

http://species-id.net/wiki/Philhygra_jarmilae

Figs 159, 333a, b, 460a-d, in Klimaszewski et al. 2011

\section{Distribution.}

\begin{tabular}{l|l}
\hline Origin & Nearctic \\
\hline Distribution & Canada: YT, NL \\
\hline YT distribution & $\begin{array}{l}\text { YUKON (NTR): Albert Creek, 60.0522, -128.9279, 3.VI.2007, soil sifting, willow } \\
\text { stand, B. Godin (LFC) 1 male. }\end{array}$ \\
\hline References & Gouix and Klimaszewski 2007, Klimaszewski et al. 2011 \\
\hline
\end{tabular}

Boreophilia herschelensis Klimaszewski \& Godin, sp. n. urn:Isid:zoobank.org:act:DD1259D2-69BE-4A73-B26F-DEA59F7F47D0 http://species-id.net/wiki/Boreophilia_herschelensis

Figs 11, 28-30, 58-61

Holotype (female). Canada, Yukon, Herschel Island, 69.5706, -138.902, 13.VI.2007, $5 \mathrm{~m}$, pitfall trap, site dominated by Carex and grasses with presence of willows (ATOR) - alluvial fan, D.G. Reid (LFC). 
Paratypes. Labeled as the holotype except: 1-3.VI.2007 (ECW) 1 male; 7.VI.2007 (ECW) 2 males; 10.VI.2007 (CNC) 1 male; 15.VI.2007 (ECW) 1 female; 17.VI.2007 (ECW) 1 male, 1 female; 19.VI.2007 (ECW) 1 female; 16.VII.2007 (LFC) 1 male, 1 female; 21.VII.2007 (ECW) 2 females; 31.VII.2007 (LFC) 1 male; 7.VI.2008 (ECW) 2 females; 7.VII.2008 (ECW) 2 females; 15.VII.2008 (ECW) 1 female; 11.VIII.2008 (ECW) 1 female.

Etymology. Named for the type locality, Herschel Island.

Diagnosis. Body narrow, subparallel, head and pronotum about the same width, elytra and abdomen slightly wider, uniformly black (Fig. 11); surface matte except for slightly glossy abdomen; pubescence fine, punctation weak and moderately dense, meshed microsculpture pronounced on forebody; head round, slightly flattened medially and with eyes about as long as postocular region of head; antennae slender, articles 4-5 slightly elongate, 6-10 subquadrate, last article elongate; pronotum transverse, narrower at base and widest at middle; elytra at suture slightly longer than or as long as pronotum; abdomen subparallel for most of its length; length $2.8-3.0 \mathrm{~mm}$ (Fig. 11). MALE: tergite 8 transverse and truncate apically (Fig. 58); sternite 8 slightly elongate and rounded apically (Fig. 59); median lobe of aedeagus with straight tubus in lateral view and with apex short and narrow (Fig. 29), dorsal aspect as illustrated (Fig. 28). FEMALE: tergite 8 transverse and truncate apically (Fig. 60); sternite 8 slightly elongate and rounded apically (Fig. 61); spermatheca S-shaped, capsule consisting of a globular apical part with a small invagination, stem sinuate (Fig. 30).

The following combination of characters distinguishes this species from other congeners: narrow, subparallel and uniformly black body, integument of forebody matte and with dense microsculpture, median lobe of aedeagus narrow apically and spermatheca S-shaped.

Distribution. This Nearctic species is known only from the type locality on Herschel Island, Yukon.

Bionomics. Adults were collected in June and July on an alluvial fan.

Comments. This species is superficially similar to B. nomensis Casey (=B. caseyiana Lohse) but differs by its uniformly black body and aedeagus with evenly narrow apical part of median lobe in lateral view.

\section{Boreophilia davidgei Klimaszewski \& Godin, sp. n.} urn:lsid:zoobank.org:act:6561B1F8-3DFD-4745-B5F3-7A3131152979 http://species-id.net/wiki/Boreophilia_davidgei

Figs 12, 31, 62, 63

Holotype (female). Canada, Yukon, EMAN Plot, Cadet Camp, 60.5951, -134.9499, 20.IX.2006, $760 \mathrm{~m}$, pitfall trap, mature white spruce and feathermoss forest, coll. EP Yukon, AJK (LFC).

Paratypes. Canada, Yukon, EMAN Plot, Cadet Camp, 60.5951, -134.9499, 29.V.2006, $760 \mathrm{~m}$, pitfall trap, mature white spruce and feathermoss forest, EP Yu- 
kon, AHW (ECW) 1 female; same data except: 15.V.2002, JF (ECW) 1 female; 12.VI.2002, EV (ECW) 1 female; 18.X.2002, FD (CNC) 2 females; 8.VII.2003, LMK31Y. LJ (ECW) 1 female; Fireweed Dr., 60.6014, -134.9387, 23.IX.2000, 772 $\mathrm{m}$, pitfall trap, mixed pine and willow forest, EP Yukon (ECW) 1 female; Whitehorse, Granger, 60.7078, -135.0971, 5.VIII.2007, 657 m, soil sifting, black spruce stand, B. Godin (ECW, LFC) 2 females; same data except: 25.VIII.2007 (LFC) 1 female; Whitehorse, Paddy's Pond, 60.7067, -135.0917, 16.IX.2007, 649 m, litter sifting, mixed aspen and white spruce forest, B. Godin (ECW) 1 female; Upper Liard, Albert Creek, 60.0522, -128.928, 8.VII.2000, 699 m, deciduous litter sifting, B. Godin (ECW, LFC) 2 females.

Etymology. Named for Douglas Davidge, biological technician (ECW), who supported the second author in his work for 20 years.

Diagnosis. Body narrow, subparallel, head narrower than pronotum, elytra and abdomen slightly wider, uniformly brown with appendages yellowish-brown and antennae yellow, or with head and abdomen dark brown and rest of body light brown (Fig. 12); surface moderately glossy; pubescence fine, punctation weak and moderately dense, meshed microsculpture pronounced on forebody; head round, slightly flattened medially and with eyes about as long as postocular region of head; antennae slender, articles 4-5 slightly elongate, 6-10 subquadrate to slightly transverse, last article elongate; pronotum transverse, widest in basal half; elytra at suture slightly longer than pronotum; abdomen broadly arcuate laterally; length 2.8-2.9 mm (Fig. 12). MALE: unknown. FEMALE: tergite 8 transverse and truncate apically (Fig. 62); sternite 8 slightly elongate and rounded apically (Fig. 63); spermatheca S-shaped, capsule elongate, stem short and sinuate (Fig. 31).

The following combination of characters distinguishes this species from other congeners: body narrow, subparallel and brown, with pronotum, elytra and legs lighter, antennae yellowish, surface of forebody moderately glossy and with dense microsculpture, and spermatheca short and S-shaped.

Distribution. This Nearctic species is known only from the type localities in the Yukon Territory.

Bionomics. Adults were collected from May to September from soil and organic litter.

Comments. This species may be easily distinguished by the unique shape of the spermatheca.

\section{Acknowledgements}

Pamela Cheers, English Editor (LFC) edited the first draft of the manuscript. Adam Brunke (University of Guelph, Ontario) corrected the first draft of this manuscript and provided many useful comments. The second author thanks the following individuals for supporting this project: Douglas Davidge (ECW) for coordinating the collection of samples in the Nisutlin Wildlife Area in conjunction with Debbie van de Wetering; his wife Denise Horwood who assisted with the collection of most of his samples; 
Donald G. Reid (Whitehorse) for the collection of specimens from Herschel Island, Elise Bolduc (Université du Québec à Rimouski) for sorting Staphylinidae from the Herschel Island samples. The collection of samples from Herschel Island was supported by grants from the Government of Canada's International Polar Year program (Indian and Northern Affairs), the Natural Sciences and Engineering Research Council of Canada, Environment Canada, and the Polar Continental Shelf Program (Natural Resources Canada), the Arctic Wildlife Observatories Linking Vulnerable EcoSystems International Polar Year project (project \#11, ArcticWOLVES) of the Centre d'études nordiques, Université Laval, Québec. We are most obliged to A. Smetana (CNC) for his comments on and significant improvements of the original manuscript.

\section{References}

Ashe JS (2000) Keys to the tribes and genera of Nearctic Aleocharinae. In: Arnett RH, Thomas MC (Eds) American Beetles, 1. Archostemata, Myxophaga, Adephaga, Polyphaga: Staphyliniformia. CRC Press LLC, Boca Raton, Florida, 299-374.

Assing V (2002) A taxonomic and phylogenetic revision of Amarochara Thomson. I. The species of the Holarctic region (Coleoptera: Staphylinidae, Aleocharinae, Oxypodini). Beiträge zur Entomologie, Keltern 52: 111-204.

Assing V (2008) The genus Calodera Mannerheim in Canada (Insecta, Coleoptera, Staphylinidae, Aleocharinae). ZooKeys 2: 203-208. doi: 10.3897/zookeys.2.6

Bernhauer M (1907) Neue Aleocharini aus Nordamerika. (Col.) (3. Stück.). Deutsche Entomologische Zeitschrift 1907: 381-405.

Buse A, Good JEG (1993) The effects of conifer forest design and management on abundance and diversity of rove beetles (Coleoptera: Staphylinidae): implications for conservation. Biological Conservation 64: 67-76. doi: 10.1016/0006-3207(93)90384-D

Campbell JM, Davies A (1991) Family Staphylinidae. Rove beetles. In: Bousquet Y (Ed) Checklist of beetles of Canada and Alaska. Publication 1861/E, Agriculture Canada, Ottawa, Ontario, 86-124.

Casey TL (1894) Coleopterological Notices. V. Annals of the New York Academy of Sciences 7: 281-606, pl. 1.

Casey TL (1910) New species of the staphylinid tribe Myrmedoniini. [pp. 1-183] In: Memoirs on the Coleoptera. I. New Era Printing Co., Lancaster, Pennsylvania, 205 pp. doi: 10.5962/bhl.title.1159

Casey TL (1911) New American species of Aleocharinae and Myllaeninae. [pp. 1-245] In: Memoirs on the Coleoptera. II. The New Era Printing Co., Lancaster, Pennsylvania, 259 pp.

Fenyes A (1910) Two new species of Aleocharinae from California. Proceedings of the Entomological Society of Washington 11[1909]: 197-199.

Fenyes A (1920) Coleoptera. Fam. Staphylinidae, subfam. Aleocharinae. In: Wytsman PA (Ed) Genera Insectorum 173(b): 111-414.

Fenyes A (1921) New genera and species of Aleocharinae with a polytomic synopsis of the tribes. Bulletin of the Museum of Comparative Zoology 65(2): 17-36. 
Gouix N, Klimaszewski J (2007) Catalogue of aleocharine rove beetles of Canada and Alaska (Coleoptera, Staphylinidae, Aleocharinae). Pensoft Series Faunistica 65. Pensoft Publishers, Sofia, Bulgaria, 165 pp.

Gusarov VI (2003) Revision of some types of North American aleocharines (Coleoptera: Staphylinidae: Aleocharinae), with synonymic notes. Zootaxa 353: 1-134.

Gusarov VI (2004) A revision of the genus Lypoglossa Fenyes, 1918 (Coleoptera: Staphylinidae: Aleocharinae). Zootaxa 747: 1-36.

Hoebeke ER (1992) Taxonomy and distribution of the athetine genus Lypoglossa Fenyes (Coleoptera: Staphylinidae: Aleocharinae) in North America, with description of a new species. Journal of the New York Entomological Society 100: 381-398.

Klimaszewski J (1984) A revision of the genus Aleochara Gravenhorst of America north of Mexico (Coleoptera: Staphylinidae, Aleocharinae). Memoirs of the Entomological Society of Canada 129: 3-211.

Klimaszewski J, Winchester NN (2002) Aleocharine rove beetles (Coleoptera Staphylinidae) of the ancient Sitka spruce forest on Vancouver Island, British Columbia, Canada. Mémoires de la Société royale belge d'Entomologie 40: 3-126.

Klimaszewski J, Sweeney J, Price J, Pelletier G (2005) Rove beetles (Coleoptera: Staphylinidae) in red spruce stands, eastern Canada: diversity, abundance, and descriptions of new species. The Canadian Entomologist 137: 1-48. doi: 10.4039/n03-123

Klimaszewski J, Pelletier G, Germain C, Work T, Hébert C (2006) Review of Oxypoda species in Canada and Alaska (Coleoptera, Staphylinidae, Aleocharinae): systematics, bionomics, and distribution. The Canadian Entomologist 138: 737-852. doi: 10.4039/n05-064

Klimaszewski J, Assing V, Majka CG, Pelletier G, Webster RP, Langor D (2007) Records of adventive aleocharine beetles (Coleoptera: Staphylinidae: Aleocharinae) found in Canada. The Canadian Entomologist 139: 54-79. doi: 10.4039/n05-105

Klimaszewski J, Godin B, Pelletier G, Savard K (2008) Six new species and records of aleocharine beetles from the Yukon and Alaska (Coleoptera: Staphylinidae: Aleocharinae). The Canadian Entomologist 140: 265-291. doi: 10.4039/n07-054

Klimaszewski J, Webster RP, Savard K (2009) Review of the rove beetle species of the subtribe Gyrophaenina Kraatz (Coleoptera, Staphylinidae) from New Brunswick, Canada: new species, provincial records and bionomic information. ZooKeys 22: 81-170. doi: 10.3897/ zookeys.22.219

Klimaszewski J, Langor D, Pelletier G, Bourdon C, Perdereau L (2011) Aleocharine beetles (Coleoptera, Staphylinidae) of the province of Newfoundland and Labrador, Canada. Pensoft Series Faunistica 98, Pensoft Publishers, Sofia, Bulgaria, 313 pp.

Leschen RAB (1993) Evolution patterns of feeding in selected Staphylinidae (Coleoptera): shift among food texture. In: Schaefer CW, Leschen RAB (Eds) Functional Morphology of Insect Feeding. Thomas Say Publications in Entomology, Entomological Society of America, Lanham, Maryland, 59-104.

Lohse GA, Smetana A (1985) Revision of the types of species of Oxypodini and Athetini (sensu Seevers) described by Mannerheim and Mäklin from North America (Coleoptera: Staphylinidae). The Coleopterists Bulletin 39: 281-300. 
Lohse GA, Klimaszewski J, Smetana A (1990) Revision of Arctic Aleocharinae of North America (Coleoptera: Staphylinidae). The Coleopterists Bulletin 44: 121-202.

Majka CG, Klimaszewski J, Lauff RF (2006) New Coleoptera records from owl nests in Nova Scotia, Canada. Zootaxa 1194: 33-47.

Majka CG, Klimaszewski J (2008a) New records of Canadian Aleocharinae (Coleoptera: Staphylinidae). In: Majka CG, Klimaszewski J (Eds) Biodiversity, Biosystematics, and Ecology of Canadian Coleoptera. ZooKeys 2: 85-114. doi: 10.3897/zookeys.2.7

Majka CG, Klimaszewski J, Lauff RF (2008b) The coastal rove beetles (Coleoptera, Staphylinidae) of Atlantic Canada: a survey and new records. ZooKeys 2: 115-150. doi: 10.3897/ zookeys. 2.2

Majka CG, Klimaszewski J (2010) Contributions to the knowledge of the Aleocharinae (Coleoptera, Staphylinidae) in the Maritime Provinces of Canada. ZooKeys 46: 15-39. doi: 10.3897/zookeys.46.413

Mäklin F (1853) [New species and notes]. In: Mannerheim, C.G. (Ed.): Dritter Nachtrag zur Kaefer-Fauna der Nord-Amerikanischen Laender des Russischen Reiches. Bulletin de la Société Impériale des Naturalistes de Moscou 26: 95-273.

Moore I, Legner EF (1975) A catalogue of the Staphylinidae of America north of Mexico (Coleoptera). University of California Division of Agricultural Sciences. Special Publication No. 3015: 1-514.

Muona J (1984) Review of Palaearctic Aleocharinae also occurring in North America (Coleoptera: Staphylinidae). Entomologica Scandinavica 15: 227-231. doi: $10.1163 / 187631284 X 00190$

Seevers CH (1951) A revision of the North American and European staphylinid beetles of the subtribe Gyrophaenae (Aleocharinae, Bolitocharini). Fieldiana Zoology 32: 657-762.

Seevers CH (1978) A generic and tribal revision of the North American Aleocharinae (Coleoptera: Staphylinidae). Fieldiana Zoology 71: i-vi, 1-289.

Smetana A (2004) Subfamily Aleocharinae Fleming, 1921 [pp. 353-494] In: Löbl I, Smetana A (Eds) Catalogue of Palaearctic Coleoptera, Vol. 2. Stenstrup, Apollo Books, 942 pp.

Webster RP, Klimaszewski J, Pelletier G, Savard K (2009) New Staphylinidae (Coleoptera) records with new collection data from New Brunswick, Canada. I. Aleocharinae. In: Majka CG, Klimaszewski J (Eds) Biodiversity, Biosystematics, and Ecology of Canadian Coleoptera II. ZooKeys 22: 171-248. doi: 10.3897/zookeys.22.152 